\title{
MicroRNA-148a-3p inhibits the proliferation of cervical cancer cells by regulating the expression levels of DNMT1 and UTF1
}

\author{
QING CHEN ${ }^{1}$, YIDONG WANG ${ }^{1}$, HUIMIN DANG $^{2}$ and XIAOLING WU ${ }^{1}$ \\ Departments of ${ }^{1}$ Obstetrics and Gynecology, and ${ }^{2}$ Integrated Traditional Chinese and Western Medicine, \\ The Second Affiliated Hospital of Xi'an Jiaotong University, Xi'an, Shaanxi 710004, P.R. China
}

Received January 31, 2021; Accepted May 21, 2021

DOI: $10.3892 / \mathrm{ol} .2021 .12878$

\begin{abstract}
MicroRNAs (miRs) serve a key role in carcinogenesis. miR-148a-3p has been demonstrated to act as a tumor suppressor in several tumors, such as epithelial ovarian cancer and esophageal cancer. However, to the best of our knowledge, the role of miR-148a-3p in cervical cancer remains unclear. In the present study, the expression levels of miR-148a-3p measured by reverse transcription-quantitative PCR were significantly decreased in cervical cancer tissues compared with that in normal cervical tissues. Furthermore, overexpression of miR-148a-3p markedly suppressed the proliferation of cervical cancer cells. The luciferase reporter assay demonstrated that DNA methyltransferase 1 (DNMT1) was the target gene of miR-148a-3p and that its expression measured by western blotting was inhibited by miR-148a-3p in cervical cancer cells. Correlation analysis highlighted that the expression levels of the undifferentiated embryonic cell transcription factor-1 (UTF1) were negatively associated with the expression levels of DNMT1 in cervical cancer tissues. Furthermore, DNMT1 knockdown increased the expression of UTF1 and decreased the methylation level of UTF1 promoter. These data demonstrated the expression levels of UTF1 were regulated by DNMT1 methylation in cervical cancer cells. Collectively, the results of the present study suggested that miR-148a-3p may inhibit the proliferation of cervical cancer cells by regulating the expression levels of DNMT1/UTF1, which provides potential therapeutic targets for cervical cancer.
\end{abstract}

\section{Introduction}

Cervical cancer is one of the most common malignant tumors to occur in women (1). An estimated 527,600 new cases of cervical cancer were diagnosed worldwide and 265,700 women succumbed to this disease in 2012 (2). The majority

Correspondence to: Dr Xiaoling Wu, Department of Obstetrics and Gynecology, The Second Affiliated Hospital of Xi'an Jiaotong University, 157 Xiwu Road, Xi'an, Shaanxi 710004, P.R. China

E-mail:wxlxjtu07@sina.com

Key words: DNA methyltransferase 1, microRNA-148a-3p, cervical cancer, methylation, cell proliferation of these cases occured in developing countries (3). Currently, recurrence, metastasis and drug resistance are the major obstacles encountered in the treatment of cervical cancer (4). Therefore, the pathogenesis of cervical cancer requires further investigations to improve current treatment options.

MicroRNAs (miRNAs/miRs) have been demonstrated to serve an important role in tumorigenesis $(5,6)$. miRNAs are a group of small, non-coding RNAs 22 nucleotides in length (7). miRNAs function as guide molecules in gene silencing and translational repression by binding to the 3'-untranslated region (3'-UTR) of their target mRNAs (8). Abnormal expression of miRNAs is closely associated with tumor initiation, progression and prognosis (9). miR-148a is a novel tumor suppressor gene, which is involved in various biological functions, including cell apoptosis, cell cycle arrest and cell senescence $(10,11)$. The expression levels of miR-148a have been reported to be dysregulated in various cancer types, such as prostate, pancreatic (12) and colorectal cancer (13). However, to the best of our knowledge, the effects and underlying molecular mechanism of miR-148a-3p in cervical cancer remain unclear. Therefore, the present study aimed to investigate the effects and the mechanism of miR-148a-3p in cervical cancer. The findings provide potential therapeutic targets for cervical cancer.

\section{Materials and methods}

Patient tissue samples. A total of 20 cervical cancer (mean \pm SD age, 56 \pm 10.05 years; age range, 39-68 years old, all female) and 8 normal cervical samples (mean \pm SD age, $53 \pm 9.13$ years; age range, 40-65 years old, all female) were collected from patients that underwent surgical resection at The Second Affiliated Hospital of Xi'an Jiaotong University (Xi'an, China) between February 2017 and February 2018. In cases with histologically confirmed cervical cancer, only patients who underwent diagnostic procedures, such as biopsy were included. Any patients with non-epithelial cervical cancer, recurrent disease and other malignancies were excluded from the present study. The normal cervical tissues were obtained from patients with uterine leiomyoma. None of the patients had received chemotherapy, immunotherapy or radiotherapy prior to specimen collection. All tissue samples were frozen in liquid nitrogen at $-80^{\circ} \mathrm{C}$ until required for further experiments. The present study was approved by the Ethics Committee of 
The Second Affiliated Hospital of Xi'an Jiaotong University (Xi'an, China) and the patients provided written informed consent prior to sample collection.

Cell lines and culture. The HeLa and SiHa human cervical cancer cell lines and human embryonic kidney cell line 293T were purchased from American Type Culture Collection and cultured in DMEM (Sigma-Aldrich; Merck KGaA) supplemented with 10\% heat-inactivated FBS (Invitrogen; Thermo Fisher Scientific, Inc.), $80 \mathrm{U} / \mathrm{ml}$ penicillin and $80 \mathrm{ug} / \mathrm{ml}$ streptomycin. The cells were maintained at $37^{\circ} \mathrm{C}$ with $5 \% \mathrm{CO}_{2}$.

Reverse transcription-quantitative PCR (RT-qPCR). Total RNA was extracted from frozen samples and cell lines using TRIzol ${ }^{\circledR}$ reagent (Invitrogen; Thermo Fisher Scientific, Inc.). RT reactions were performed using the PrimeScript RT reagent kit (Takara Bio, Inc.) according to the manufacturer's protocol. Subsequently, qPCR was performed using the SYBR-Green Master mix (Takara Bio, Inc.) according to the manufacturer's protocol. GAPDH and U6 spliceosomal RNA were used as an internal control for the quantification of mRNAs and miRNAs, respectively. The primer sequences are shown in Table I. The thermocycling conditions were as follows: Pre-denaturation at $50^{\circ} \mathrm{C}$ for 2 min, denaturation at $95^{\circ} \mathrm{C}$ for $10 \mathrm{~min}$, annealing at $95^{\circ} \mathrm{C}$ for $30 \mathrm{sec}$ and extension at $60^{\circ} \mathrm{C}$ for $30 \mathrm{sec}(40$ cycles). The relative gene expression was quantified using the $2^{-\Delta \Delta C \mathrm{C}}$ method (14).

Cell Counting Kit-8 (CCK-8) assay. Cell proliferation was measured using a CCK- 8 assay (Beyotime Institute of Biotechnology). Briefly, $1 \times 10^{3}$ cells/well were cultured in 96-well plates and assessed the following day. The assessment was carried out for 6 days in total. At the same time point on 2, 4, 6 days, $10 \mu$ 1 CCK-8 solution was added to each well and the samples were incubated for $4 \mathrm{~h}$ at $37^{\circ} \mathrm{C}$. The absorbance was measured at a wavelength of $450 \mathrm{~nm}$ using a plate reader. Each experiment was performed in triplicate.

Western blot analysis. Total protein was extracted from frozen samples and cell lines using RIPA lysis buffer (Beyotime Institute of Biotechnology). The protein concentration was estimated using a BCA assay and $20 \mu \mathrm{g}$ protein/lane was separated via $10 \%$ SDS-PAGE, and then transferred onto PVDF membranes (MilliporeSigma). The membranes were blocked with 5\% skimmed milk suspended in TBST at room temperature for $2 \mathrm{~h}$. The membranes were incubated with primary antibodies against UTF1 (1:100; cat. no. ab65453; Abcam); DNMT1 (1:200; cat. no. SC-20701; Santa Cruz Biotechnology, Inc.) or GAPDH (1:1,000; cat. no. AB-P-R 001; Hangzhou Xianzhi Biological Co., Ltd.) at $4^{\circ} \mathrm{C}$ overnight. Following the primary antibody incubation, the membranes were incubated with horseradish peroxidase-conjugated secondary antibodies (1:10,000; cat. no. BA1054; Wuhan Boster Biological Technology Co. Ltd.) at $37^{\circ} \mathrm{C}$ for $2 \mathrm{~h}$. The membranes were briefly incubated with an enhanced chemiluminescence reagent (MilliporeSigma) at room temperature for $2 \mathrm{~min}$ and visualized using X-ray films. GAPDH was used to normalize the expression of the genes. Protein level was quantified using Quantity One software v.4.6, (Bio-Rad Laboratories, Inc.).
Cell transfection. miR-148a-3p mimic (5'-UCAGUGCAC UACAGAACUUUGU-3'), miRNA mimic control (5'-TTC TCCGAACGTGTCACGT-3'), DNMT1-short hairpin (sh) RNA plasmid expression vector (pGPU6/GFP/Neo, 5'-GGAU GAGUCCAUCAAGGAATT-3') and control-shRNA (5'-UUC UCCGAACGUGUCACGUTT-3') were purchased from Shanghai GenePharma Co., Ltd. Cells were incubated $\left(1 \times 10^{5}\right)$ in a 6-well plate for at $37^{\circ} \mathrm{C}$ for $24 \mathrm{~h}$ before transfection and transfected with either miR-148a-3p mimic, control mimic, DNMT1-shRNA and control-shRNA at a final concentration of $50 \mathrm{nM}$ using Lipofectamine ${ }^{\circledast} 2000$ transfection reagent (Invitrogen; Thermo Fisher Scientific, Inc.) at $37^{\circ} \mathrm{C}$ for $5 \mathrm{~h}$ according to the manufacturer's protocol. After $48 \mathrm{~h}$, the cells were used for subsequent experiments.

Luciferase reporter assay. $293 \mathrm{~T}$ cells were seeded into a 24 well plate at a density of $5 \times 10^{4}$ cells/well. Following incubation at $37^{\circ} \mathrm{C}$ for $24 \mathrm{~h}$, a wild type or mutated DNMT1 3'-UTR luciferase reporter vector (Promega Corporation), combined with miR-148a-3p mimics (5'-UCAGUGCACUACAGA ACUUUGU-3'; Shanghai GenePharma Co., Ltd.) or miRNA mimic control (5'-TTCTCCGAACGTGTCACGT-3', Shanghai GenePharma Co., Ltd.), were transfected into the cells at a final concentration of $20 \mathrm{nM}$ using a Vigofect transfection reagent [Weiglas Biotechnology (Beijing) Co., Ltd.] according to the manufacturer's protocol. At $48 \mathrm{~h}$ post-transfection, the firefly and Renilla luciferase activities were detected using the Dual-Luciferase Reporter assay system (Promega Corporation). Renilla luciferase activity was used as the internal control.

Bisulfite sequencing. Bisulfite sequencing was carried out as previously described (15). Genomic DNA was extracted from $\mathrm{SiHa}$ and HeLa cells using the Universal Genomic DNA Extraction kit (cat. no. DV811A; Takara Bio, Inc.) according to the manufacturer's protocol. Genomic DNA $(250 \mathrm{ng})$ of each sample was bisulfite converted using EpiTect Bisulfite kit (cat. no. 59104; Qiagen, Inc.) according to the manufacturer's protocol. A 360 bp segment (nucleotides -977 to -617 , transcriptional start site, +1 ) from bisulfate-modified DNA was amplified using MSP DNA polymerase (TIANGEN Biotech Co., Ltd.) with the following primer sequences: Forward, 5'-TGATTAGAGTAGGGATGGAAAG-3' and reverse, 5'-TAC AACCAACATCCCTAAAAA-3'. The thermocycling conditions were as follows: 1 cycle at $95^{\circ} \mathrm{C}$ for $10 \mathrm{~min}$, followed by amplification for 40 cycles at $95^{\circ} \mathrm{C}$ for $30 \mathrm{sec}, 60^{\circ} \mathrm{C}$ for $30 \mathrm{sec}$ and $72^{\circ} \mathrm{C}$ for $30 \mathrm{sec}$ and final extension at $72^{\circ} \mathrm{C}$ for $10 \mathrm{~min}$. The PCR products were recovered and purified by $1.0 \%$ agarose gel electrophoresis and quantified using the ImageJ software v.1.53a (National Institutes of Health), then subcloned by TA cloning using the pEASY-T1 Cloning kit (cat. no. CT101-01; TransGen Biotech Co., Ltd.) and then transformed into Escherichia coli strain DH5a (Invitrogen; Thermo Fisher Scientific Inc.) using standard procedures. Recombinant plasmids positive for inserts of correct size (559 bp) were identified by colony PCR with Taq DNA polymerase (Takara Bio, Inc.). At least 10 positive inserted clones were selected and sequenced by Wuhan Biofavor Biotech Service Co., Ltd. using Sanger sequencing method (POP-7 ${ }^{\mathrm{TM}}$ Polymer for 3730/3730x1 DNA Analyzers, cat. no. 4332241; Thermo Fisher Scientific Inc.) 
Table I. Primer sequences.

\begin{tabular}{lll}
\hline Name & Primer & Sequence (5'-3') \\
\hline GAPDH & Forward & TCAAGAAGGTGGTGAAGCAGG \\
& Reverse & TCAAAGGTGGAGGAGTGGGT \\
DNMT1 & Forward & TACCACGCAGACATCAACCT \\
& Reverse & GCCCTTCCCTTTGTTTCCAG \\
UTF1 & Forward & ATGGGGCTGCTGGGCGACAACG \\
miR-148a-3p & Reverse & GGGGAGGCGTCCGCAGACTTCG \\
& Forward & TGCGCTCAGTGCACTACAGAAC \\
U6 & Reverse & CCAGTGCAGGGTCCGAGGTATT \\
& Forward & CGCTTCGGCAGCACATATAC \\
\hline
\end{tabular}

DNMT1, DNA methyltransferase 1; miR-148a-3p, microRNA-148a-3p; UTF1, undifferentiated embryonic cell transcription factor-1.

with M13 primers (M13 forward, 5'-GTAAAACGACGGCCA GT-3' and reverse, 5'-CAGGAAACAGCTATGAC-3'). The quality of processed samples was estimated according to the optical density (OD) 260/280 ratio; the ratio between $1.8-2.0$ meet the experimental requirements. The OD 260/280 ratio of DNA was estimated using a microspectrophotometer and the concentration of DNA was calculated according to the formula: Total DNA concentration $(\mu \mathrm{g} / \mu \mathrm{l})=\mathrm{OD} 260 \times 50 \times 200 \times 10^{-3}$. The concentration requirement: $>50 \mathrm{ng} / \mu \mathrm{l}$. The methylation density was quantified using BiQ Analyzer software v.2.0 (16) (Max Planck Institute Informatik).

Statistical analysis. Statistical analysis was performed using SPSS version 16.0 software (SPSS, Inc.). Data are presented as the mean \pm SD. An independent sample unpaired t-test and one-way ANOVA followed by Tukey's post hoc test were used for group comparisons. Correlation analysis was performed using Pearson's correlation analysis. The experiments were performed in triplicate and repeated three times independently. $\mathrm{P}<0.05$ was considered to indicate a statistically significant difference.

\section{Results}

Expression levels of miR-148a-3p in cervical cancer. To explore the role of miR-148a-3p in cervical cancer, its expression levels were assessed in cervical cancer and normal cervical tissues by RT-qPCR analysis. miR-148a-3p expression was significantly decreased in cervical cancer tissues compared with in normal cervical tissues $(\mathrm{P}<0.01$; Fig. 1). These data suggested that miR-148a-3p may be associated with the progression of cervical cancer.

miR-148a-3p inhibits the proliferation of cervical cancer cells. To assess the effects of miR-148a-3p on the proliferation of cervical cancer cells, miR-148a-3p mimics were successfully transfected into HeLa and SiHa cells (Fig. 2A and B) and cell proliferation was evaluated using a CCK- 8 assay. The cell proliferation curve revealed that the viability of miR-148a-3p overexpressing HeLa and SiHa cells were significantly

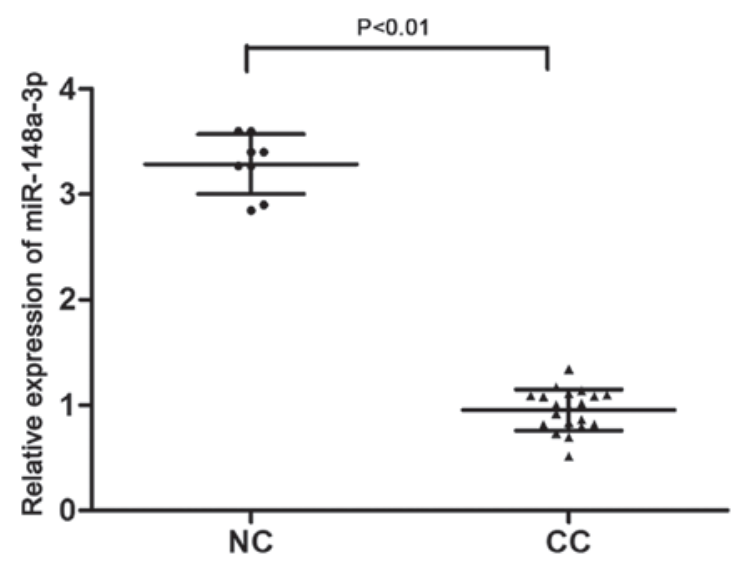

Figure 1. Expression levels of miR-148a-3p in NC and CC tissues. CC, cervical cancer; NC, normal cervix; miR-148a-3p, microRNA-148a-3p.

decreased compared with the control mimics $(\mathrm{P}<0.01$; Fig. 2C and D). These results demonstrated that miR-148a-3p inhibited the proliferation of cervical cancer cells.

miR-148a-3p inhibits DNMT1 expression by targeting the 3'-UTR. The protein expression levels of DNMT1 were significantly increased in cervical cancer tissues compared with in normal cervical tissues $(\mathrm{P}<0.001$; Fig. 3A and $\mathrm{B})$. In addition, the mRNA expression levels of DNMT1 in cervical cancer were significantly higher than those in normal cervical tissues $(\mathrm{P}<0.01$; Fig. 3C). Correlation analysis indicated that the expression levels of DNMT1 were negatively correlated with miR-148a-3p expression $(\mathrm{P}<0.01$; Fig. 3D), suggesting that DNMT1 expression may be regulated by miR-148a-3p. Using bioinformatics analysis, it was identified that DNMT1 was a potential target gene of miR-148a-3p (Fig. 4A). To verify this hypothesis, luciferase reporter vectors containing the potential binding sequences of the 3'-UTR of DNMT1 (wt and mut) were constructed and co-transfected with miR-148a-3p or mimic control into 293T cells (Fig. 4A). The luciferase activity levels in the wt DNMT1 + miR-148a-3p group were significantly decreased compared with those of 
A

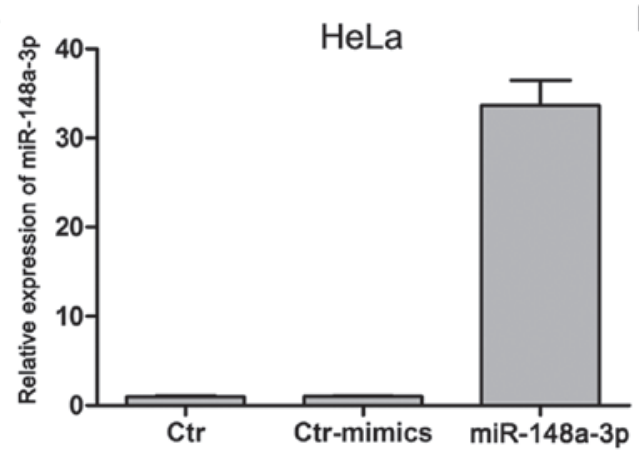

C

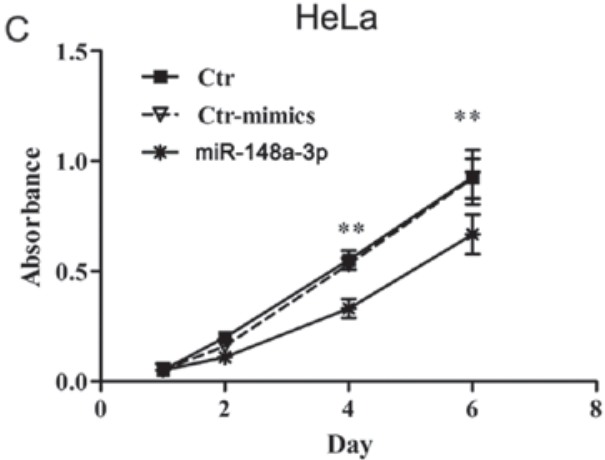

B

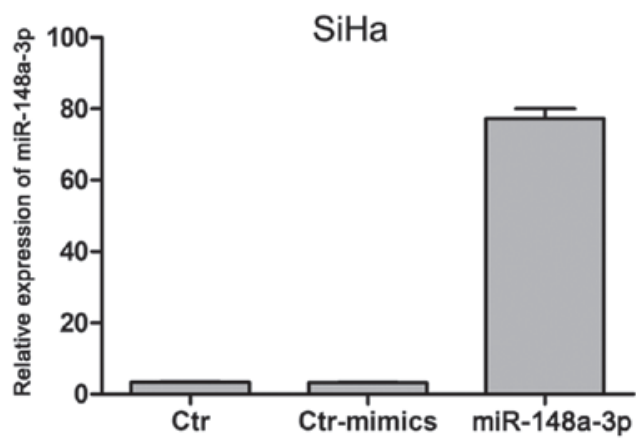

$\mathrm{SiHa}$

D

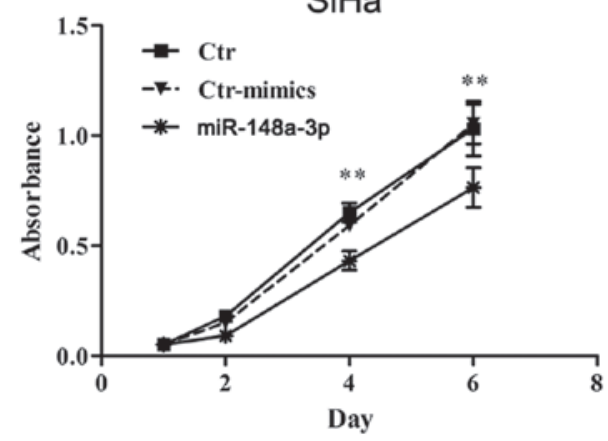

Figure 2. miR-148a-3p inhibits the proliferation of HeLa and SiHa cervical cancer cells. The expression of miR-148a-3p in (A) HeLa and (B) SiHa cells by transfection with miR-148a-3p and control mimics. (C) miR-148a-3p expressing HeLa and (D) SiHa cell proliferation was inhibited compared with the control mimics. ${ }^{* *} \mathrm{P}<0.01$ vs. Ctr-mimics. Ctr, control; miR-148a-3p, microRNA-148a-3p.

A

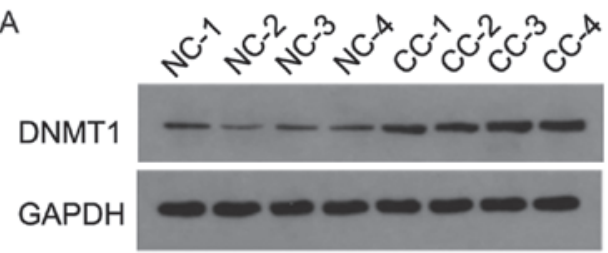

C

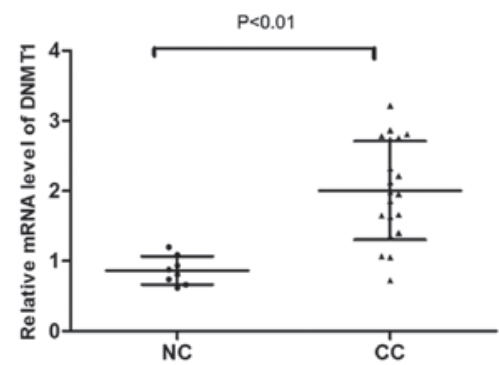

B

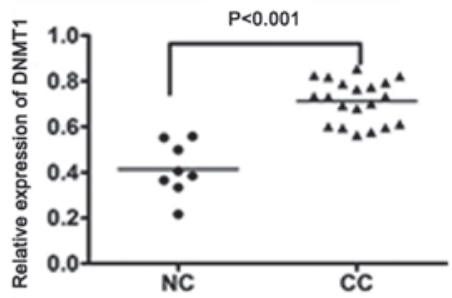

D

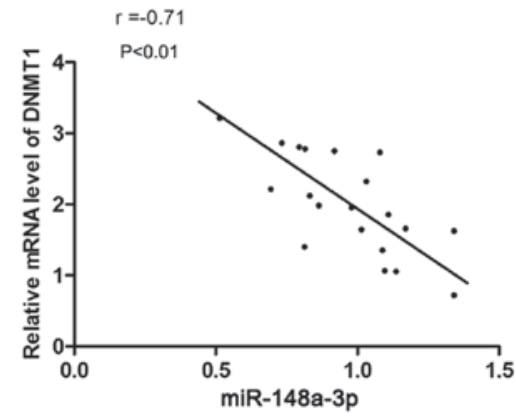

Figure 3. DNMT1 expression is inversely correlated with miR-148a-3p expression in CC. (A) Detection of DNMT1 expression in NC and CC tissues was performed by western blot analysis. (B) Expression levels of DNMT1 in NC and CC tissues were normalized to GAPDH. (C) Assessment of the mRNA expression levels of DNMT1 in NC and CC tissues. (D) DNMT1 expression was negatively correlated with miR-148a-3p expression in CC tissues. CC, cervical cancer; DNMT1, DNA methyltransferase 1; miR-148a-3p, microRNA-148a-3p; NC, normal cervix.

the wt DNMT1 + control mimic group, whereas the luciferase activity in the mut DNMT1 + miR-148a-3p group was not significantly altered compared with that of the mut DNMT1 + control mimic group (Fig. 4B). These findings demonstrated that miR-148a-3p targeted the 3'-UTR of DNMT1.
In addition, to further verify the regulatory effect of miR-148a-3p on DNMT1 expression, the expression of DNMT1 in miR-148a-3p overexpressing HeLa and SiHa cells were measured. DNMT1 mRNA (Fig. 5A and B) and protein expression levels (Fig. 5C-F) were significantly decreased in 
A

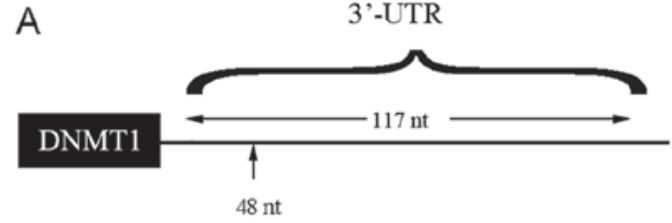

miR-148a-3p 3' UGUUUCAAGACAUCACGUGACU 5'

wt DNMT1 3'-UTR 5' CAGGAAUCCCCAACAUGCACUUGA 3' mut DNMT1 3'-UTR 5' CAGGAAUCCCCAACAUCAGCUGA 3'
B

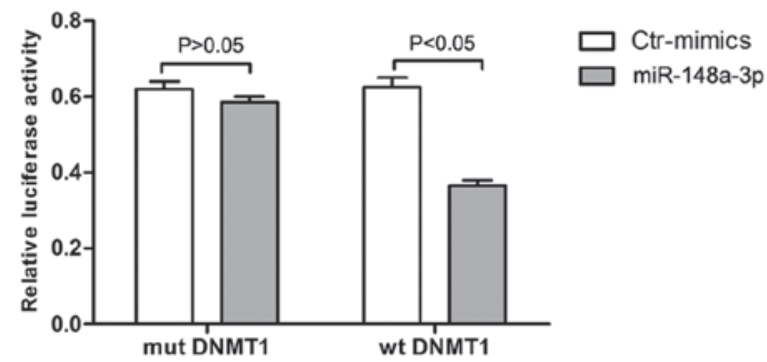

Figure 4. miR-148a-3p targets the 3'-UTR of DNMT1. (A) Schematic diagram of the miR-148a-3p putative targeting site in wt and mut sequences of DNMT1. (B) Relative luciferase activity in 293T cells co-transfected with miR-148a-3p or Ctr-mimics and luciferase reporter vector constructs. The wt or mut sequences of DNMT1 3'-UTR were used. Ctr, control; DNMT1, DNA methyltransferase 1; miR-148a-3p, microRNA-148a; mut, mutant; 3'-UTR; 3'-untranslated region; wt, wild-type.

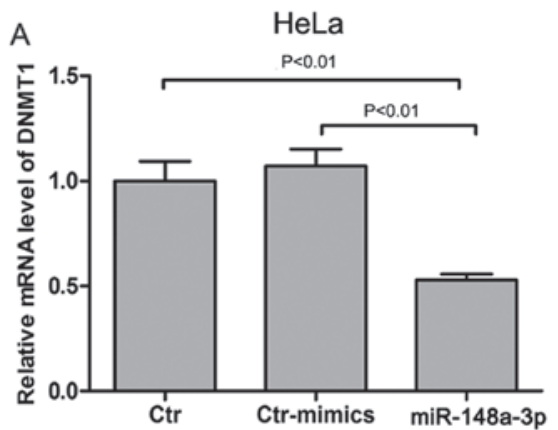

B
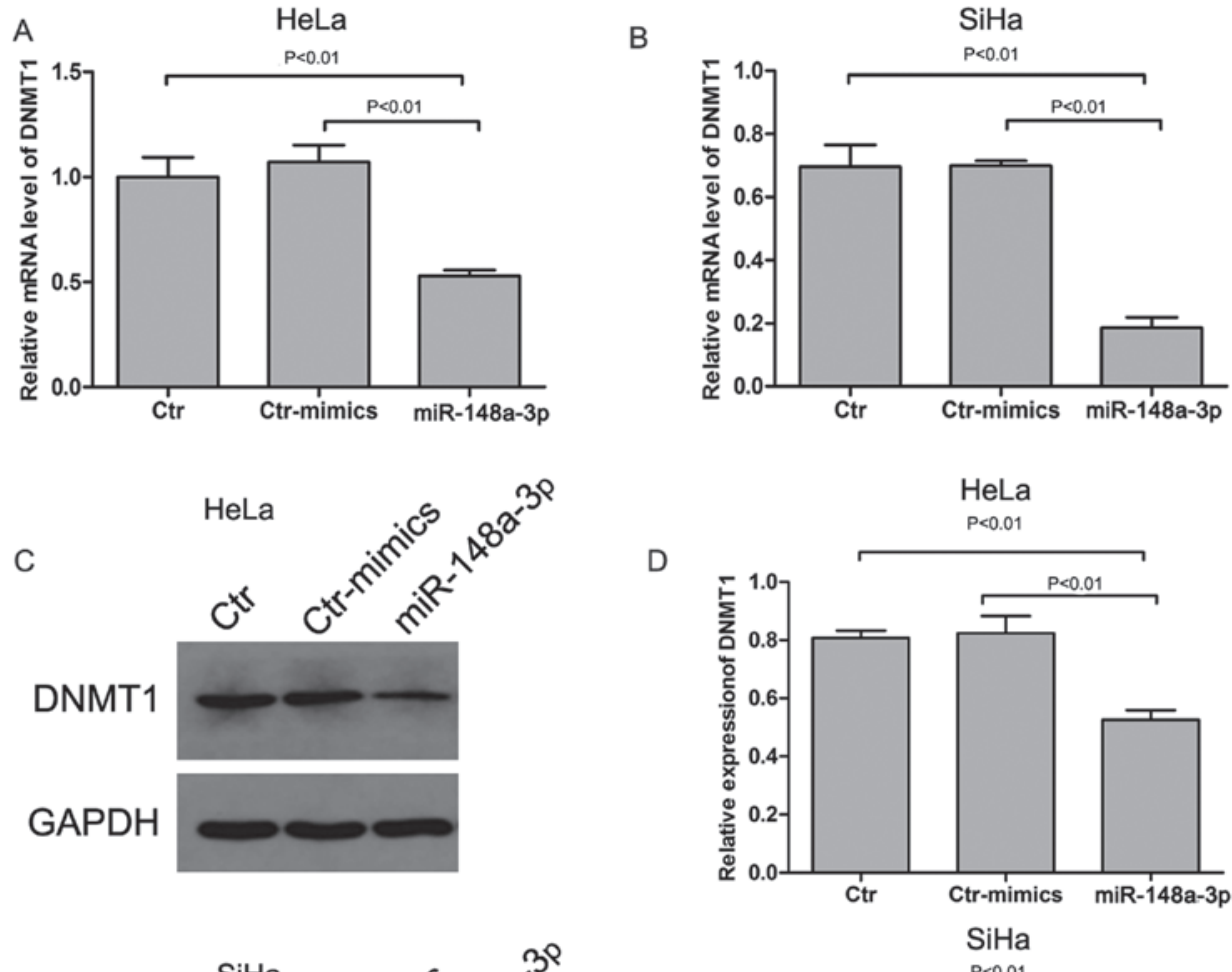

$\mathrm{E}$

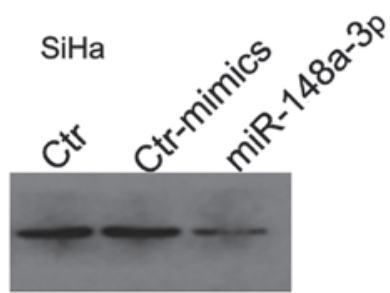

$\mathrm{F}$

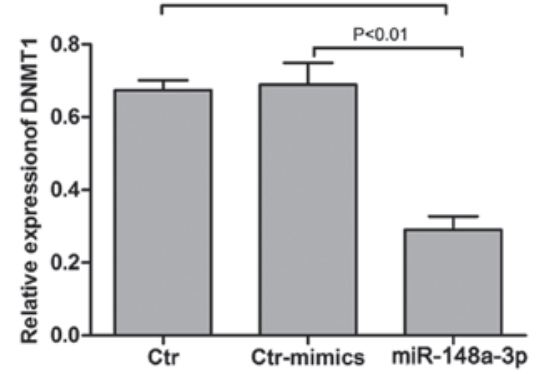

Figure 5. miR-148a-3p inhibits DNMT1 expression in HeLa and SiHa cells. (A) DNMT1 mRNA expression were measured in HeLa cells transfected with miR-148a-3p or control mimics by RT-qPCR. (B) DNMT1 mRNA expression were measured in SiHa cells transfected with miR-148a-3p or control mimics by RT-qPCR. (C) Protein expression of DNMT1 in HeLa cells transfected with miR-148a-3p or control mimics were measured by western blotting. (D) Relative quantitative analysis of protein expression of DNMT1 in HeLa cells transfected with miR-148a-3p or control mimics. (E) Protein expression of DNMT1 in $\mathrm{SiHa}$ cells transfected with miR-148a-3p or control mimics were measured by western blotting. (F) Relative quantitative analysis of protein expression of DNMT1 in SiHa cells transfected with miR-148a-3p or control mimics . Ctr, control; DNMT1, DNA methyltransferase 1; miR-148a-3p, microRNA-148a-3p; RT-q, reverse transcription-quantitative.

miR-148a-3p-overexpressing HeLa and SiHa cells compared with those of the control mimics $(\mathrm{P}<0.01)$. These data further demonstrated that miR-148a-3p regulated DNMT1 expression by targeting its 3 '-UTR in cervical cancer cells. 
A

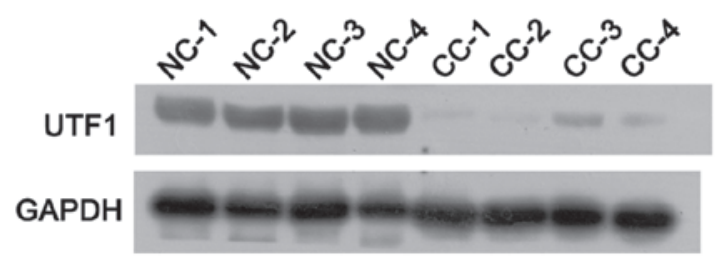

C

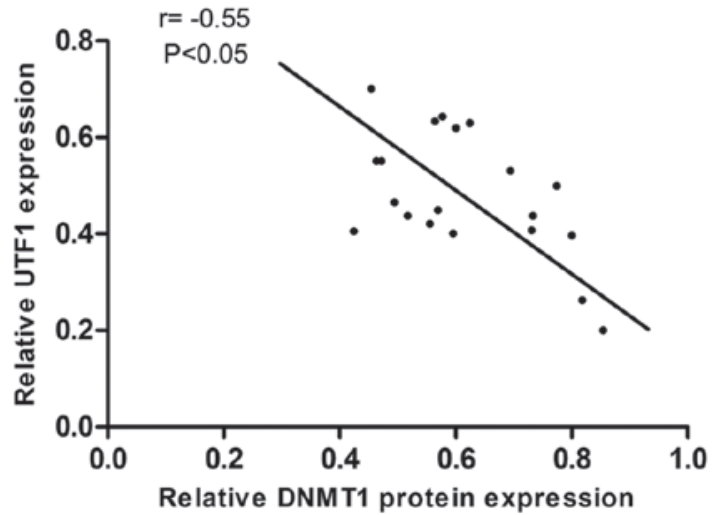

B

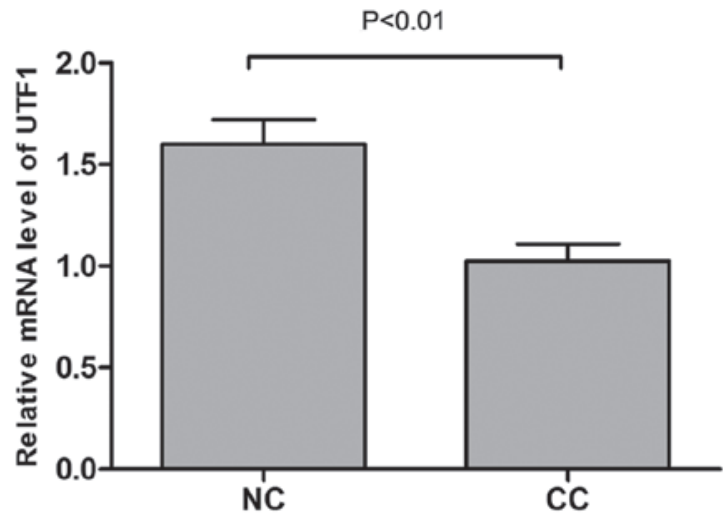

D

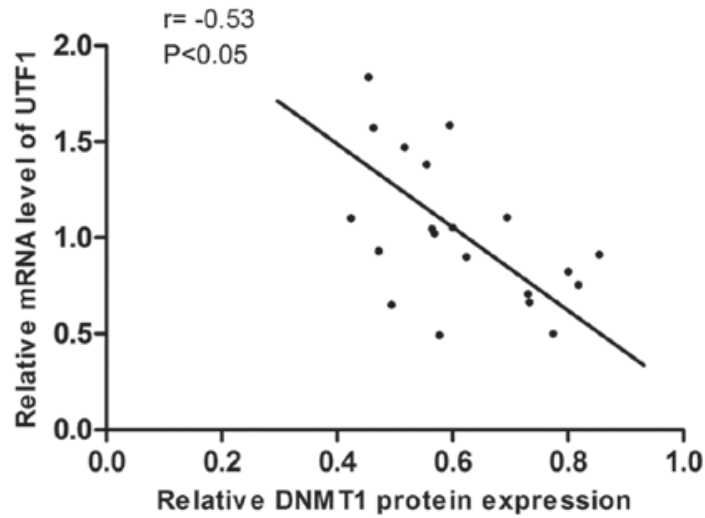

Figure 6. UTF1 expression is negatively correlated with DNMT1 expression in CC tissues. (A) UTF1 expression in CC and NC tissues were measured by western blotting. (B) UTF1 mRNA expression in CC and NC tissues were measured by RT-qPCR. (C) UTF1 protein expression exhibited a negative correlation with DNMT1 protein expression in CC. (D) UTF1 mRNA expression was negatively correlated with DNMT1 protein expression in CC. CC, cervical cancer; DNMT1, DNA methyltransferase 1; NC, normal cervix; UTF1, undifferentiated embryonic cell transcription factor-; RT-q, reverse transcription-quantitative.

DNMT1 regulates the expression levels of UTF1 via methylation in cervical cancer. In our previous study, UTF1 was demonstrated to serve an important tumor suppressive role in cervical carcinogenesis (15). However, UTF1 is highly methylated in cervical cancer (15). Based on the important role of DNMT1 in DNA methylation (17), it was hypothesized that UTF1 expression may be regulated by DNMT1 in cervical cancer. Correlation analysis indicated that the protein $(\mathrm{r}=-0.55$; $\mathrm{P}<0.05$, Fig. 6A) and mRNA expression levels $(\mathrm{r}=-0.53$; $\mathrm{P}<0.05$, Fig. $6 \mathrm{~B}$ ) of UTF1 were negatively correlated with the protein expression levels of the DNMT1 in cervical cancer. Additional experiments indicated that DNMT1 knockdown led to a significant increase in the mRNA and protein expression levels of UTF1 in HeLa and SiHa cells $(\mathrm{P}<0.01$ or $\mathrm{P}<0.05$; Fig. 7). Furthermore, compared with the control knockdown group, the methylation levels of the UTF1 promoter were significantly attenuated following DNMT1 knockdown $(\mathrm{P}<0.05$; Fig. 8). These findings demonstrated the important role of DNMT1 in the regulation of UTF1 expression.

\section{Discussion}

Numerous miRNAs have been reported to serve important roles in the pathogenesis of tumors by regulating cell proliferation, apoptosis and invasion $(18,19)$. It has been reported that miR-148a exhibits antitumor effects in various cancer types including gastric, colorectal, pancreatic, liver and breast cancers $(13,20)$. In the present study, the data indicated that miR-148a-3p expression was reduced in cervical cancer tissues compared with in normal cervical tissues. Furthermore, miR-148a-3p overexpression significantly inhibited the proliferation of HeLa and $\mathrm{SiHa}$ cells. These findings suggested that miR-148a-3p exerted inhibitory effects in cervical cancer, which was consistent with a previous study that demonstrated that miR-148a acts as a tumor suppressor gene in colorectal cancer (21).

The antiproliferative mechanism of action of miR-148a-3p was investigated by identifying its target gene, DNMT1, using bioinformatic analysis. Furthermore, a previous study has reported that $\mathrm{miR}-148 \mathrm{a}-3 \mathrm{p}$ directly represses the expression levels of DNMT1 in human colon cancer cells (22). Therefore, DNMT1 expression was analyzed in cervical cancer tissues and a negative correlation was observed between the expression levels of DNMT1 and miR-148a-3p, implying that miR-148a-3p may regulate DNMT1 expression in cervical cancer. In addition, the present study demonstrated that miR-148a-3p targeted the 3'-UTR of DNMT1. Additional experiments demonstrated that overexpression of miR-148a-3p inhibited the protein and mRNA expression of DNMT1 in HeLa and SiHa cells. Collectively, these data demonstrated that miR-148a-3p regulated DNMT1 expression by targeting its 3'-UTR sequence in cervical cancer.

UTF1 is a stem cell-associated transcription factor, which serves a critical role in cell differentiation and 

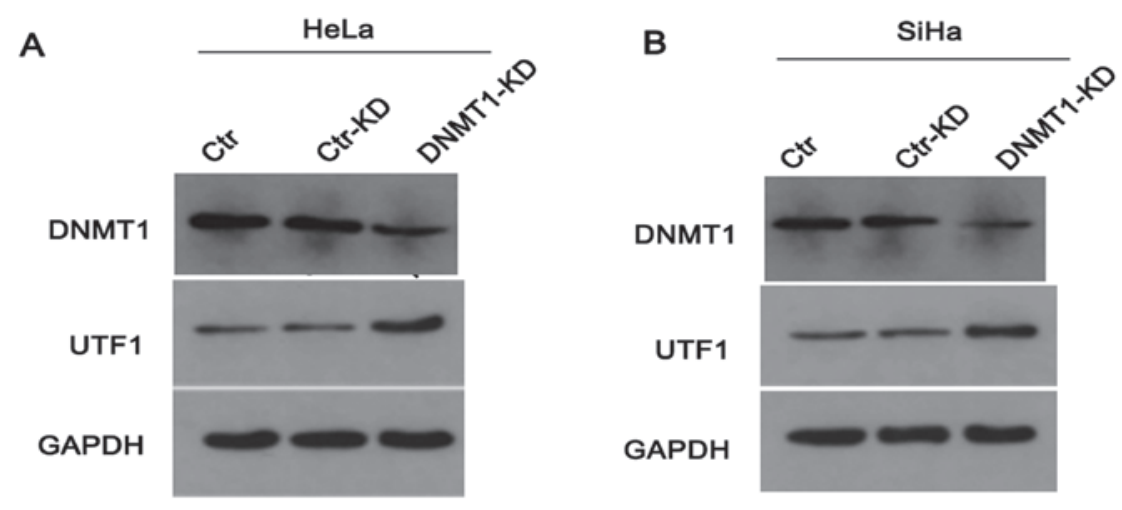

C

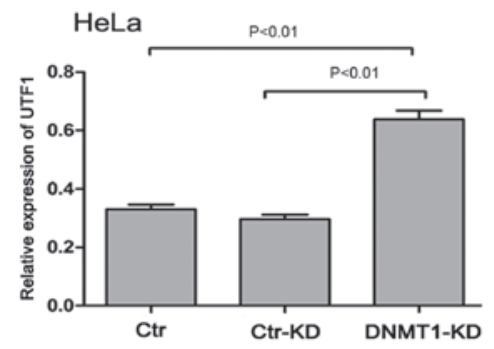

D

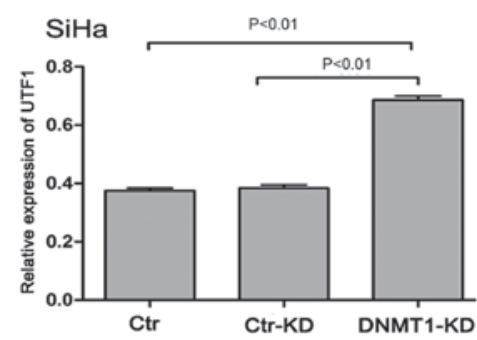

$\mathrm{E}$
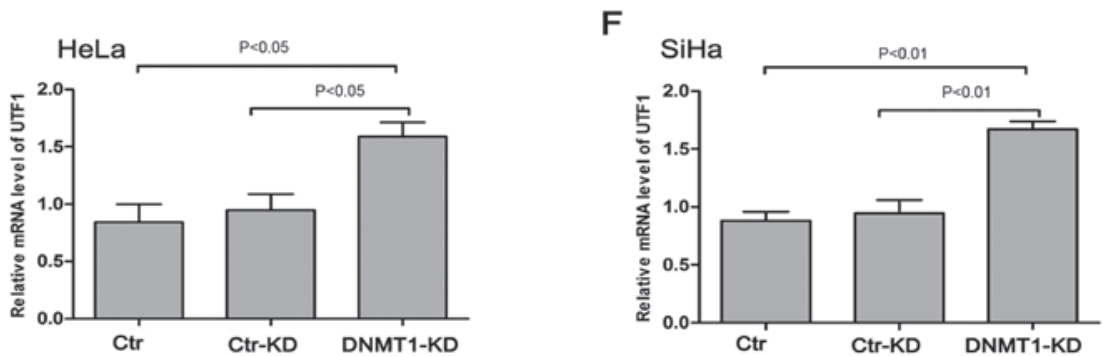

Figure 7. DNMT1 knockdown increases the expression levels of UTF1 in HeLa and SiHa cells. DNMT1 knockdown increased the protein expression levels of UTF1 in (A) HeLa and (B) SiHa cells. The expression levels of UTF1 in (C) HeLa and (D) SiHa cells were normalized to those of GAPDH. DNMT1 knockdown increased the mRNA expression levels of UTF1 in (E) HeLa and (F) SiHa cells. Ctr, control; DNMT1, DNA methyltransferase 1; KD, knockdown; UTF1, undifferentiated embryonic cell transcription factor-1.
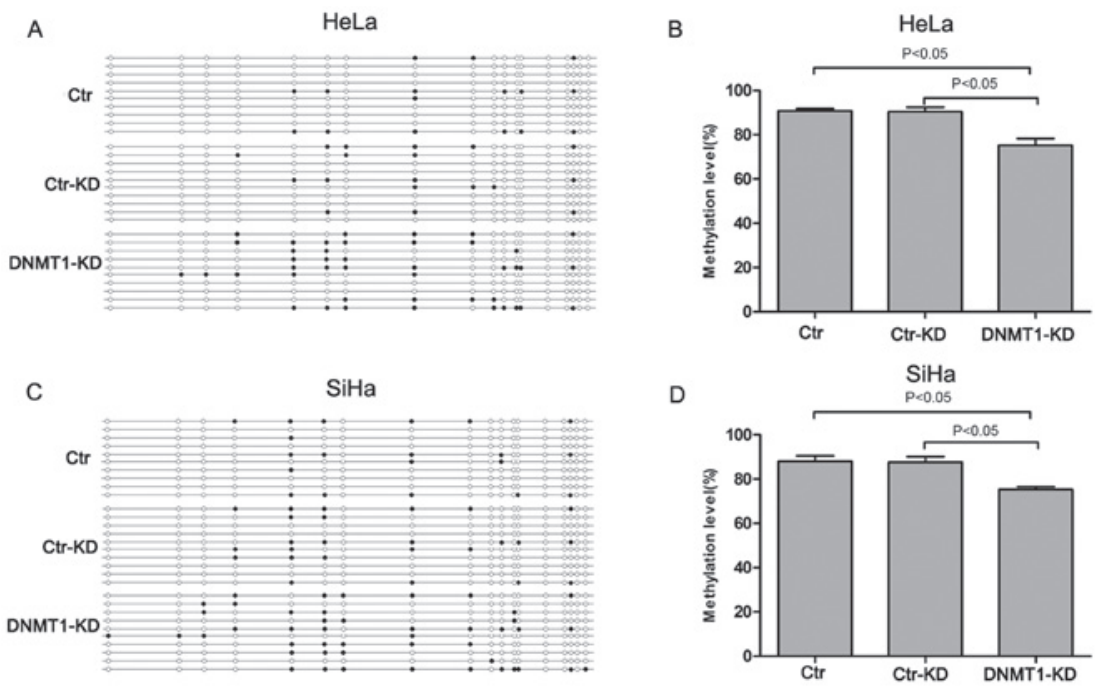

Figure 8. DNMT1 knockdown reduces the methylation levels of UTF1. (A) Methylation level of the UTF1 promoter was measured in DNMT1 knockdown HeLa cells. (B) Percentage of methylation of UTF1 promoter in DNMT1 knockdown HeLa cells was lower compared with the control. (C) Methylation level of the UTF1 promoter was measured in DNMT1 knockdown SiHa cells. (D) Percentage of methylation of UTF1 promoter in DNMT1 knockdown HeLa cells was lower compared with the control. Methylation analysis included all $18 \mathrm{CpG}$ dinucleotides within the 100 base pair amplicon. The results are shown using 10 independent clones for each group. White circles indicate methylated regions, black circles indicate non-methylated regions. Ctr, control; DNMT1, DNA methyltransferase 1; KD, knockdown; UTF1, undifferentiated embryonic cell transcription factor-1. 
development (23). In our previous study, UTF1 functioned as a tumor suppressor gene and its expression was downregulated in cervical cancer (15). In addition, the promoter region of UTF1 was hypermethylated in cervical cancer $(15,24)$. DNMT enzymes typically mediate global hypermethylation of the genome (25). DNMT1 is an important member of the DNMT superfamily (26). Notably, In the present study, DNMT1 was highly expressed in cervical cancer and the association between the expression levels of UTF1 and DNMT1 was analyzed in cervical cancer tissues. UTF1 expression was negatively correlated with DNMT1 expression, implying that the latter may regulate UTF1 expression in cervical cancer. DNMT1 knockdown significantly increased the expression levels of UTF1 in HeLa and SiHa cells, which demonstrated that DNMT1 regulated UTF1 expression in cervical cancer. The modification of DNA methylation is considered the main pattern of epigenetic regulation (27). DNMT1 serves a key role in DNA methylation (28). It has been demonstrated that the overexpression of DNMT1 increases DNA methylation (29). In the present study, methylation analysis indicated that DNMT1 knockdown significantly reduced the methylation levels of the UTF1 promoter in cervical cancer cells. These findings are consistent with a previous report that demonstrated that promoter hypermethylation contributes to the decreased expression of tumor suppressor genes (30).

In summary, the results of the present study demonstrated that miR-148a-3p inhibited the proliferation of cervical cancer cells. The mechanism of action was associated with the regulation of the expression of DNMT1 and UTF1, which may provide potential therapeutic targets for cervical cancer.

\section{Acknowledgements}

Not applicable.

\section{Funding}

The present study was supported by the National Natural Science Foundation of China (grant no. 81702578).

\section{Availability of data and materials}

The datasets used and/or analyzed during the current study are available from the corresponding author on reasonable request.

\section{Authors' contributions}

QC and YW performed the experiments and data analysis. XW conceived and designed the study. QC and HD confirmed the authenticity of all the raw data. XW and HD reviewed and revised the manuscript for important intellectual content. HD participated in data analysis and draft writing. All authors read and approved the final manuscript.

\section{Ethics approval and consent to participate}

The present study was approved by the Ethics Committee of The Second Affiliated Hospital of Xi'an Jiaotong University (approval no. 2017-113; Xi'an, China). All the patients signed written informed consent prior to participation in the present study.

\section{Patient consent for publication}

Not applicable.

\section{Competing interests}

The authors declare that they have no competing interests.

\section{References}

1. Small W Jr, Bacon MA, Bajaj A, Chuang LT, Fisher BJ, Harkenrider MM, Jhingran A, Kitchener HC, Mileshkin LR, Viswanathan AN and Gaffney DK: Cervical cancer: A global health crisis. Cancer 123: 2404-2412, 2017.

2. Torre LA, Bray F, Siegel RL, Ferlay J, Lortet-Tieulent J and Jemal A: Global cancer statistics, 2012. CA Cancer J Clin 65: 87-108, 2015.

3. Wang J, Lv H, Xue Z, Wang L and Bai Z: Temporal trends of common female malignances on breast, cervical, and ovarian cancer mortality in japan, republic of Korea, and Singapore: Application of the age-period-cohort model. Biomed Res Int 2018: 5307459, 2018.

4. Gupta S, Kumar P and Das BC: HPV: Molecular pathways and targets. Curr Probl Cancer 42: 161-174, 2018.

5. Drusco A and Croce CM: MicroRNAs and cancer: A long story for short RNAs. Adv Cancer Res 135: 1-24, 2017.

6. Fanini F and Fabbri M: Cancer-derived exosomic microRNAs shape the immune system within the tumor microenvironment: State of the art. Semin Cell Dev Biol 67: 23-28, 2017.

7. Costello A, Lao N, Clynes M and Barron N: Conditional knockdown of endogenous MicroRNAs in $\mathrm{CHO}$ cells using TET-ON-SanDI sponge vectors. Methods Mol Biol 1603: 87-100, 2017.

8. Höck $\mathrm{J}$ and Meister G: The Argonaute protein family. Genome Biol 9: 210, 2008.

9. Lin X, Xiaoqin H, Jiayu C, Li F, Yue L and Ximing X: Long non-coding RNA miR143HG predicts good prognosis and inhibits tumor multiplication and metastasis by suppressing mitogen-activated protein kinase and Wnt signaling pathways in hepatocellular carcinoma. Hepatol Res 49: 902-918, 2019.

10. Zhang M, Wang C and Mu L: Mir-148a induces apoptosis by upregulating bim expression in gastric cancer cells. Int J Clin Exp Med 10: 2791-2799, 2017.

11. Liu J, Si L and Tian H: MicroRNA-148a inhibits cell proliferation and cell cycle progression in lung adenocarcinoma via directly targeting transcription factor E2F3. Exp Ther Med 16: 5400-5409, 2018.

12. Xiao WD, Ao J, Huang Z, Lin SR, Peng L and Li Y: The effects of up-regulation of miR-148a on the expression of anti-oncogene ppENK, p16 and RASSF1A in pancreatic carcinoma AsPC-1 cells. Pancreatology 16 (Suppl): S15, 2016.

13. Zhang H, Li Y, Huang Q, Ren X, Hu H, Sheng H and Lai M: MiR-148a promotes apoptosis by targeting $\mathrm{Bcl}-2$ in colorectal cancer. Cell Death Differ 18: 1702-1710, 2011.

14. Livak KJ and Schmittgen TD: Analysis of relative gene expression data using real-time quantitative PCR and the 2(-Delta Delta C(T)) method. Methods 25: 402-408, 2001.

15. Wu XL and Zheng PS: Undifferentiated embryonic cell transcription factor-1 (UTF1) inhibits the growth of cervical cancer cells by transactivating p27 Kip1. Carcinogenesis 34: 1660-1668, 2013.

16. Bock C, Reither S, Mikeska T, Paulsen M, Walter J and Lengauer T: BiQ Analyzer: Visualization and quality control for DNA methylation data from bisulfite sequencing. Bioinformatics 21: 4067-4068, 2005.

17. Han M, Li J, Cao Y, Huang Y, Li W, Zhu H, Zhao Q, Han JJ, Wu Q, Li J, et al: A role for LSH in facilitating DNA methylation by DNMT1 through enhancing UHRF1 chromatin association. Nucleic Acids Res 48: 12116-12134, 2020.

18. Wei Y, Peng S, Wu M, Sachidanandam R, Tu Z, Zhang S, Falce C, Sobie EA, Lebeche D and Zhao Y: Multifaceted roles of miR-1s in repressing the fetal gene program in the heart. Cell Res 24: 278-292, 2014.

19. Yu X and Li Z: The role of microRNAs expression in laryngeal cancer. Oncotarget 6: 23297-23305, 2015.

20. Li Y, Deng X, Zeng X and Peng X: The role of Mir-148a in cancer. J Cancer 7: 1233-1241, 2016. 
21. Zhao W, Zheng J, Wei G, Yang K, Wang G and Sun X: miR-148a inhibits cell proliferation and migration through targeting ErbB3 in colorectal cancer. Oncol Lett 18: 2530-2536, 2019.

22. Zuo J, Xia J, Ju F, Yan J, Zhu A, Jin S, Shan T and Zhou H: MicroRNA-148a can regulate runt-related transcription factor 3 gene expression via modulation of DNA methyltransferase 1 in gastric cancer. Mol Cells 35: 313-319, 2013.

23. Pantazis G, Harter PN, Capper D, Kohlhof P, Mittelbronn M and Schittenhelm J: The embryonic stem cell factor UTF1 serves as a reliable diagnostic marker for germinomas. Pathology 46 : 225-229, 2014.

24. Okuda A, Fukushima A, Nishimoto M, Orimo A, Yamagishi T, Nabeshima Y, Kuro-o M, Nabeshima Yi, Boon K, Keaveney M, et al: UTF1, a novel transcriptional coactivator expressed in pluripotent embryonic stem cells and extra-embryonic cells. EMBO J 17: 2019-2032, 1998.

25. Jin B and Robertson KD: DNA Methyltransferases, DNA damage repair, and cancer. Adv Exp Med Biol 754: 3-29, 2013.

26. Foltz G, Yoon JG, Lee H, Ryken TC, Sibenaller Z, Ehrich M, Hood L and Madan A: DNA methyltransferase-mediated transcriptional silencing in malignant glioma: A combined whole-genome microarray and promoter array analysis. Oncogene 28: 2667-2677, 2009.
27. Scopa CD, Zolota V and Kourea HP: Targeted pathways in breast cancer: Molecular and protein markers guiding therapeutic decisions. Curr Mol Pharmacol 7: 4-21, 2014.

28. Kumar R and Rao DN: Role of DNA Methyltransferases in epigenetic regulation in bacteria. Subcell Biochem 61: 81-102, 2013.

29. Subramaniam D, Thombre R, Dhar A and Anant S: DNA Methyltransferases: A novel target for prevention and therapy. Front Oncol 4: 80-80, 2014

30. You Y, Yang W, Wang Z, Zhu H, Li H, Lin C and Ran Y: Promoter hypermethylation contributes to the frequent suppression of theCDK10 gene in human nasopharyngeal carcinomas. Cell Oncol (Dordr) 36: 323-331, 2013. 